\title{
Using the P03 precession model
}

\author{
Patrick T. Wallace ${ }^{1}$ and Nicole Capitaine ${ }^{2}$ \\ ${ }^{1}$ CCLRC/, Rutherford Appleton Laboratory, Didcot, UK \\ email: ptw@star.rl.ac.uk
}

${ }^{2}$ SYRTE/UMR8630, Observatoire de Paris, 61 Avenue de l'Observatoire, F-75014 Paris, France e-mail: n.capitaine@obspm.fr

Abstract. We discuss aspects of using the P03 precession model.

Keywords. reference systems, ephemerides, astrometry

The IAU 2000 precession model comprised the existing Lieske et al. (1977) model plus rate corrections.

Though a good fit to existing VLBI observations, the IUA 2000 model model is not consistent with dynamical theory, and so the IAU Working Group on precession and the ecliptic recommended (Hilton et al. 2006) that it be replaced by the 'P03' model of Capitaine et al. (2003).

P03 provides improved models for both the equator and the ecliptic, and also includes parameterized provision for future adjustment to match new determinations of properties of the non-rigid Earth.

Practical use of the new model involves choices, and various ways have been studied (Capitaine \& Wallace 2006) of generating the directions of the celestial intermediate pole and origin (CIP, CIO), from which the usual rotation matrices can be obtained.

From a wide range of possible procedures we have selected two that target different classes of application, typified by the SOFA software and the IERS Conventions respectively.

These procedures achieve a high standard of consistency, both internal and mutual, as well as being efficient and versatile. One is based on the Fukushima-Williams precessionnutation angles, the other on series for the CIP coordinates.

Both use the CIO locator $s$, and both deliver the full range of products, supporting classical equinox/GST methods in addition to the CIO/ERA 'new paradigm'.

\section{References}

Capitaine, N., Wallace, P. T., \& Chapront, J. 2003, A\& $A, 412,567$

Capitaine, N., \& Wallace, P. T. 2006, A\&BA, 450, 855

Hilton, J. L., Capitaine, N., Chapront, J., Ferrandiz, J. M., Fienga, A., Fukushima, T., Getino, J., Mathews, P., Simon, J.-L., Soffel, M., Vondrák, J., Wallace, P., \& Williams, J. 2006, Celest. Mech., 94, 351

Lieske, J. H., Lederle, T., Fricke, W., Morando, B. 1977, A\&A, 58, 1 